\title{
SAFOD Phase III Core Sampling and Data Management at the Gulf Coast Repository
}

\author{
by Bradley Weymer, John Firth, Phil Rumford, Frederick Chester, \\ Judith Chester, and David Lockner
}

\section{Introduction}

The San Andreas Fault Observatory at Depth (SAFOD) project is yielding new insight into the San Andreas Fault (Zoback et al., 2010; Zoback et al., this issue). SAFOD drilling started in 2002 with a pilot hole, and proceeded with three phrases of drilling and coring during the summers of 2004, 2005, and 2007 (Fig. 1). One key component of the project is curation, sampling, and documentation of SAFOD core usage at the Integrated Ocean Drilling Program's (IODP) Gulf Coast Repository (GCR) at Texas A\&M University. We present here the milestones accomplished over the past two years of sampling Phase III core at the GCR.

\section{Research Themes}

Several research themes rely heavily on SAFOD core samples. These are focused on understanding the structure, composition, and a variety of physical and mechanical properties of the San Andreas Fault. Structural studies are concerned with characterizing the geometry of the fault zone, the distribution of shear displacement, the process by which rocks are deformed, and the microscopic features that record past occurrences of earthquake slip and creeping deforma-

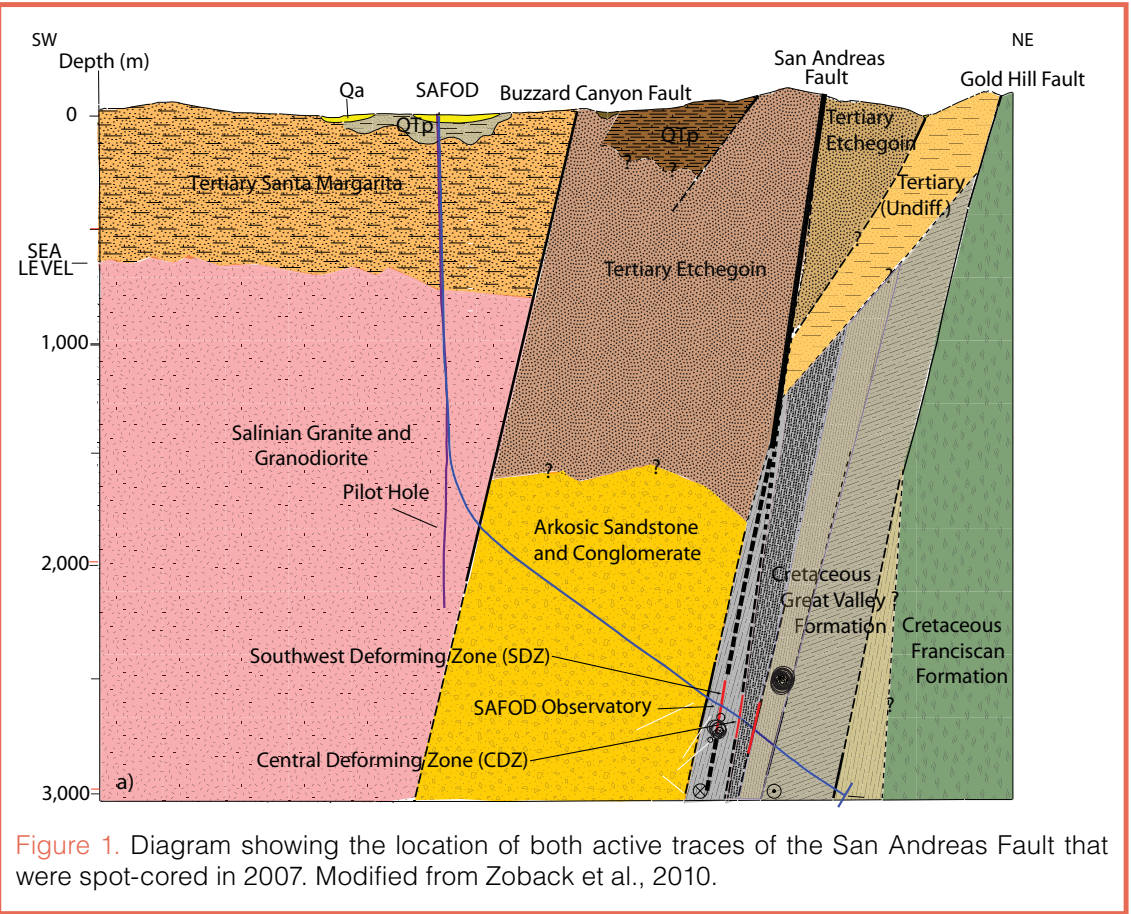

tion. Core samples are ideal for structural study at macroscopic and microscopic scales, using non-destructive techniques such as mapping the surface of the core and X-ray computed tomography (CT scanning) to image the interior of the core, as well as destructive techniques such as cutting and polishing small sub-samples for optical and electron microscopy.

Compositional studies focus on the elemental, chemical, and mineral content of the fault zone to understand the processes and conditions which occur during faulting, such as rates of chemical reactions between minerals and pore fluids, the origin of the rock and fluids in the fault, and the pressure and temperature at depth. These studies involve a variety of subsample processing techniques and analytical instruments such as X-ray diffraction and fluorescence, electron microprobe, and mass spectrometers.

Laboratory experimentation with core samples is an important way to quantify a host of physical and mechanical properties that are important to faulting processes. Sub-samples are used to determine thermal conductivity, porosity and permeability, and seismic velocity within the fault. Mechanical testing explores the fracture, friction, and flow properties of the fault rocks in the actively deforming portions of the fault zone.

\section{Inter-Laboratory Comparison Project}

The main objective of the InterLaboratory Comparison Project was to run tests on standardized sample materials to provide a baseline for the comparison of results from experiments conducted on SAFOD Phase III core samples (Lockner et al., 2009). A total of eighteen laboratories from nine countries conducted the following tests: intact rock strength, frictional strength, permeability, electrical resistivity, ultrasonic wave speed, thermal conductivity, and related properties such as porosity and density. Standardized protocols were established, and test samples were distributed to participating laboratories in the fall of 2008 . Friction tests were conducted on crushed and sieved samples of 


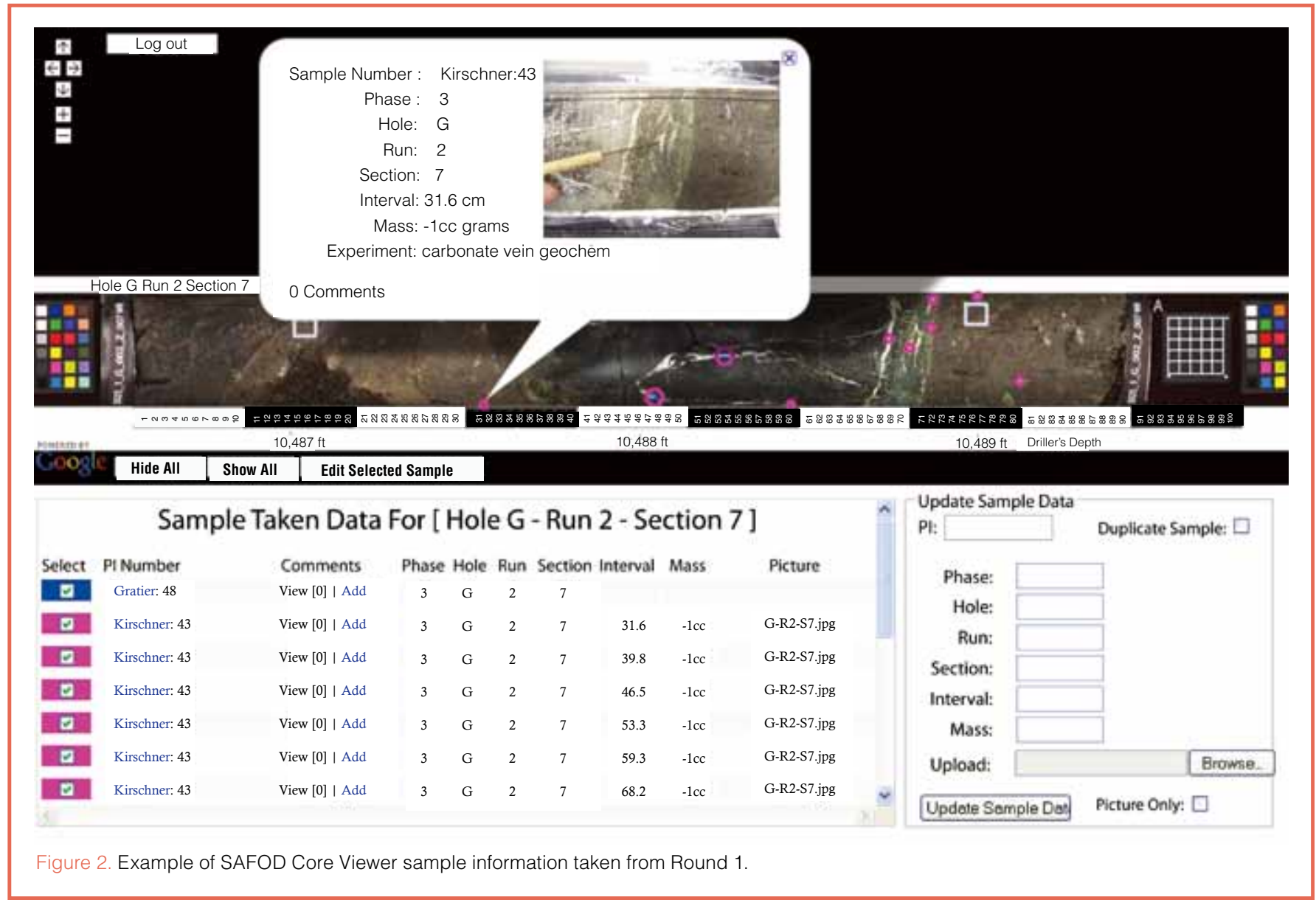

quartz, granite, talc, a quartz/smectite mixture, and SAFOD cuttings. Other physical property measurements were conducted on marble, granite, and three types of sandstones. Preliminary results of the inter-lab comparisons were discussed at the EarthScope meeting in Boise, Idaho in 2009 (Lockner et al., 2009), and further results were presented at the AGU 2010 Fall Meeting.

\section{SAFOD Sampling at the Gulf Coast Repository}

After the arrival of Phase III cores, the GCR assumed full curatorial responsibilities of all SAFOD samples (Phases 1 to 3). All SAFOD sampling follows the guidelines outlined by the SAFOD Sample Policy. This requires Principal Investigators (PIs) to submit all analytical data and metadata to the GCR for placement on the SAFOD web site in a timely manner. Currently, data are available to the public via the SAFOD Core Viewer.

The first cycle of sample requests (Round 1) were for 790 samples from twenty-eight PI groups comprising ninetyeight scientists from around the world. The approved sample distribution was 190 samples for twenty PI groups, comprising fifty-eight scientists. Round 1 sampling started on 28 June 2008 and was completed on 17 December 2008. After the completion of Round 1 sampling, the SAFOD Sample Committee (SSC) began accepting requests for Round 2.
Round 2 requests were for 344 samples from thirteen PI groups, comprising thirty-nine scientists, and all of them were approved. About $20 \%$ of the requesting PIs from Round 2 were from institutions outside the U.S. Round 2 sampling commenced on 21 July 2009 and remains a work in progress. To date, a total of 164 (as of December 2010) samples have been taken.

\section{The SAFOD Core Viewer}

A major component of the SAFOD project involved the creation and maintenance of an interactive, online core sample database and administration tool by staff at EarthScope/UNAVCO (University NAVstar COnsortium), with design input originally from Charley Weiland at Stanford University and now the IODP-GCR curatorial staff. Building upon the flexibility of Google Maps API, the SAFOD Core Viewer serves as a repository for all information related to the project from sample inventories and photo libraries to data storage. The Core Viewer has three views for each core section: (1) Samples Requested, (2) Samples Approved, and (3) Samples Taken. The Core Viewer provides the following functions (Fig. 2):

1. It allows all interested PI groups to submit their sample requests to be reviewed online by the SSC. 
2. It provides the GCR curatorial staff the ability to view all approved sample requests and aids in the sampling process.

3. After samples are taken, any information pertaining to each sample, such as the type of experiment, expected results, pictures, and subsequent data, can be uploaded into the Core Viewer (Figs. 3A, 3B).

4. After review, data are also uploaded and made available on the public Core Viewer.

\section{Acknowledgements}

We thank the SAFOD Co-PI's Mark Zoback, Stephen Hickman, and William Ellsworth, as well as the support of Charley Weiland, for their guidance and support during the sampling and data management phase of the project. A special thanks to Brian Blackman, Michael Jackson, and Adrian Borsa at UNAVCO for the development and continued improvement of the Core Viewer. We also thank Kaye Shedlock and Greg Anderson at NSF, and the SAFOD Sample Committee.
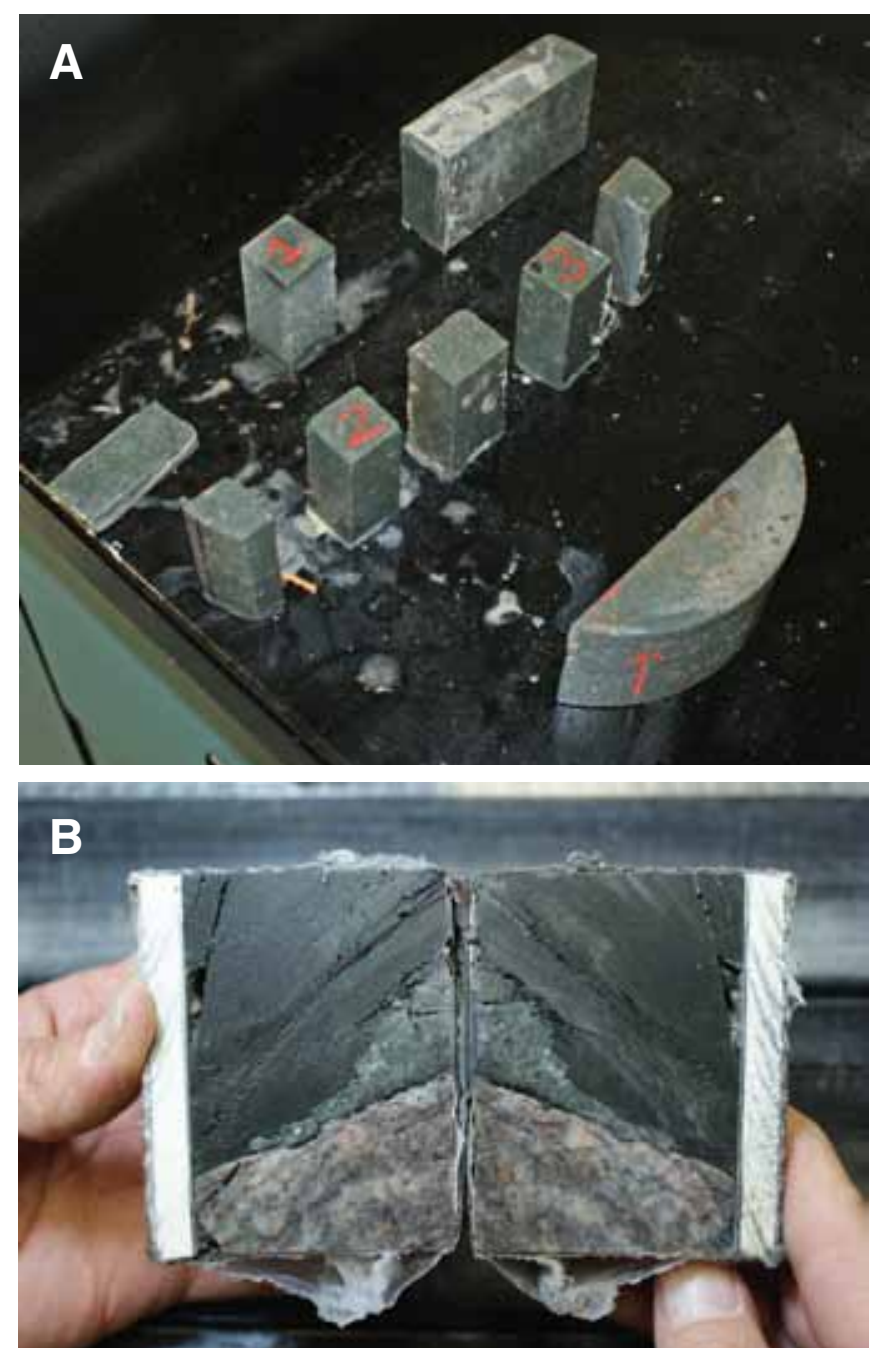

Figure 3. [A] Image of precise cuts for friction studies during Round 1 that documents the sampling process and was uploaded into the Core Viewer. [B] Example of Round 2 image within the SAFOD Core Viewer. Section E-R1-S5, showing a fault contact between dark grayish-black siltstone and grayish-red pebbly sandstone.

\section{References}

Lockner, D., Marone, C., and Saffer, D., 2009. SAFOD inter-laboratory comparisons - a progress report [poster presented at the EarthScope 2009 meeting in Boise, Idaho, 12-15 May 2009].

Zoback, M., Hickman, S., and Ellsworth, W., 2010. Scientific drilling into the San Andreas Fault zone. Eos, Trans. AGU, 91(22):197-204, doi:10.1029/2010EO220001.

\section{Authors}

Bradley Weymer, SAFOD Curatorial Specialist and Graduate Assistant Researcher, Integrated Ocean Drilling Program and SAFOD, Texas A\&M University, 1000 Discovery Drive, College Station, TX 77845-9547, U.S.A, e-mail: weymer@iodp.tamu.edu.

John Firth, Curator, and Phil Rumford, GCR Superintendent, Integrated Ocean Drilling Program and SAFOD, Texas A\&M University, 1000 Discovery Drive, College Station, TX 77845-9547, U.S.A.

Frederick M. Chester, Professor, and Judith S. Chester, Associate Professor, Department of Geology and Geophysics, TAMU, Center for Tectonophysics and Department of Geology \& Geophysics, Texas A\&M University, College Station, TX 77843-3115, U.S.A.

David Lockner, U.S. Geological Survey, Menlo Park, U.S. Geological Survey Earthquake Science Center, 345 Middlefield Road, MS/977Menlo Park, CA 94025, U.S.A.

\section{Related Web Links}

http://safod.icdp-online.org

http://www.earthscope.org/publications

http://www.earthscope.org/observatories/safod

http://www.earthscope.org/data/safod_core_viewer http://www.earthscope.org/es_doc/safod/SAFOD_Core_ Sample_Distribution.pdf http://www.earthscope.org/data/safod_core_samples

\section{Photo Credit}

Fig. 3: Photos by Bradley Weymer, IODP and SAFOD, Texas A\&M University 